\title{
Loss of CIC promotes mitotic dysregulation and chromosome segregation defects.
}

Suganthi Chittaranjan ${ }^{1}$, Jungeun Song ${ }^{1}$, Susanna Y. Chan ${ }^{1}$, Stephen Dongsoo Lee ${ }^{1}$, Shiekh Tanveer Ahmad ${ }^{2,3,4}$, William Brothers ${ }^{1}$, Richard D. Corbett ${ }^{1}$, Alessia Gagliardi ${ }^{1}$, Amy Lum $^{5}$, Annie Moradian ${ }^{6}$, Stephen Pleasance ${ }^{1}$, Robin Coope ${ }^{1}$, J Gregory Cairncross ${ }^{2,7}$, Stephen Yip ${ }^{5,8}$, Emma Laks ${ }^{5,8}$, Samuel A.J.R. Aparicio ${ }^{5,8}$, Jennifer A. Chan ${ }^{2,3,4}$, Christopher S. Hughes ${ }^{1}$, Gregg B. Morin ${ }^{1,9}$, Veronique G. LeBlanc ${ }^{1}$, Marco A. Marra ${ }^{1,9 *}$

\section{Affiliations}

${ }^{1}$ Canada's Michael Smith Genome Sciences Centre, BC Cancer, Vancouver, Canada

${ }^{2}$ Arnie Charbonneau Cancer Institute, University of Calgary, Calgary, Canada

${ }^{3}$ Department of Pathology \& Laboratory Medicine, University of Calgary, Calgary, Canada

${ }^{4}$ Alberta Children's Hospital Research Institute, University of Calgary, Calgary, Canada

${ }^{5}$ Department of Molecular Oncology, BC Cancer, Vancouver, Canada

${ }^{6}$ California Institute of Technology, Pasadena, USA

${ }^{7}$ Department of Clinical Neurosciences, University of Calgary, Calgary, Canada

${ }^{8}$ Department of Pathology and Laboratory Medicine, University of British Columbia, Vancouver, Canada

${ }^{9}$ Department of Medical Genetics, University of British Columbia, Vancouver, Canada

\section{Corresponding author}

Marco A. Marra

Genome Sciences Centre

BC Cancer

675 West 10th Avenue,

Vancouver, BC

Canada V5Z 1L3

E-mail: mmarra@bcgsc.ca

604-675-8162 


\begin{abstract}
Background: CIC is a transcriptional repressor inactivated by loss-of-function mutations in several cancer types, including gliomas, lung cancers, and gastric adenocarcinomas. CIC alterations and/or loss of CIC activity have been associated with poorer outcomes and more aggressive phenotypes across cancer types, which is consistent with the notion that CIC functions as a tumour suppressor across a wide range of contexts.

Results: Using mammalian cells lacking functional CIC, we found that CIC deficiency was associated with chromosome segregation (CS) defects, resulting in chromosomal instability and aneuploidy. These CS defects were associated with transcriptional dysregulation of spindle assembly checkpoint and cell cycle regulators. We also identified novel CIC interacting proteins, including core members of the SWI/SNF complex, and showed that they cooperatively regulated the expression of genes involved in cell cycle regulation. Finally, we showed that loss of CIC and ARID1A cooperatively increased CS defects and reduced cell viability.

Conclusions: Our study ascribes a novel role to CIC as an important regulator of the cell cycle and demonstrates that loss of CIC can lead to chromosomal instability and aneuploidy in human and murine cells through defects in CS, providing insight into the underlying mechanisms of CIC's increasingly apparent role as a "pan-cancer" tumour suppressor.
\end{abstract}

\title{
Keywords
}

CIC, SWI/SNF complex, tumour suppressor, spindle assembly checkpoint, cell cycle, chromosome segregation, aneuploidy, transcription, cancer 


\section{Introduction}

Capicua (CIC) is a transcriptional repressor that is mutated or rearranged in in several cancer types, including undifferentiated small round cell sarcomas ${ }^{1}(\sim 60 \%$ frequency), oligodendrogliomas $^{2,3}(\sim 50-80 \%)$, gastric adenocarcinomas ${ }^{4}(\sim 9 \%)$, endometrial carcinomas ${ }^{5}$ $(\sim 7 \%)$, and melanomas $(\sim 8 \%)^{6,7}$. CIC loss has also been implicated in prostate cancer ${ }^{8}$, lung cancer $^{9}$, and T-cell lymphoblastic leukemia/lymphoma ${ }^{10,11}$. CIC alterations and/or loss of CIC activity have been associated with inferior outcomes ${ }^{12-15}$ and with aggressive phenotypes such as increased metastatic ability ${ }^{9}$ and resistance to MAPK inhibitors ${ }^{16,17}$, indicating that CIC likely functions as a tumour suppressor. Though functional studies of CIC are relatively limited in mammalian models, recent reports have shown that these oncogenic phenotypes appear to be due, at least in part, to de-repression of one or more of CIC's known target genes - which include the ETS transcription factors ETV1, ETV4, and ETV5 (ETV1/4/5) - upon loss of CIC ${ }^{8-10}$.

Chromosome instability (CIN) is a process that leads to whole- or partial-chromosome gains and losses and that results in aneuploidy, a cellular genetic state that is considered a hallmark of cancer ${ }^{18}$. Common mechanisms of aneuploid and polyploid cell formation (i.e. cells that contain more than two complete sets of chromosomes) include cytokinesis failure, which can for example be due to defects in cytokinetic proteins ${ }^{19}$, errors in chromosome segregation $(\mathrm{CS})^{20}$, prolonged arrests at the spindle assembly checkpoint $(\mathrm{SAC})^{21,22}$, DNA replication errors ${ }^{23}$, and cell-cell fusion ${ }^{24}$.

Here, we describe a novel function of mammalian CIC in regulating cell cycle progression and CS, and show that loss of CIC is associated with CS defects and CIN, resulting in aneuploidy. We also find that CIC interacts with core members of the SWI/SNF complex, and 
show that together they function to regulate the transcription of genes involved in cell cycle regulation and the $\mathrm{SAC}$.

\section{Results}

\section{Nuclear CIC is subject to dynamic spatial and temporal re-distribution during the cell cycle.}

To examine the spatial dynamics of nuclear CIC, we performed immunofluorescence (IF) assays in two neural cell lines: an immortalized normal human astrocyte line $\left(\mathrm{NHA}^{25}\right.$; Figure 1a), and the $\mathrm{HOG}^{26}$ line (Supplementary Figure 1). We observed that CIC was dynamically redistributed over the course of the cell cycle in both cell lines. As shown in Figure 1a, CIC was found throughout the nucleus during interphase, but appeared to be excluded from condensed chromosomes at metaphase and telophase. During early cytokinesis, CIC foci appeared at decondensing chromosomes during initiation of nuclear envelope reassembly, and increasingly accumulated until completion of cytokinesis (Figures 1a and Supplementary Figure 1, arrowheads). Upon completion of nuclear envelope assembly, CIC was observed throughout the nucleus.

We confirmed this observation using an N-terminal-FLAG-tagged CIC-S construct (F-CICS) introduced into an isogenic $C I C$ knockout $\left(C I C^{\mathrm{KO}}\right)$ cell line derived from HEK293 $\mathrm{A}^{27}$ (hereafter referred to as $\mathrm{HEK}^{\mathrm{F}-\mathrm{CIC}-\mathrm{S}}$ ). Ectopically expressed F-CIC-S displayed similar localization dynamics as endogenous CIC (Supplementary Figure 1), indicating that both CIC isoforms were dynamically re-localized during mitotic phases, thus raising the possibility that some CIC functions may be related to the cell cycle. 


\section{Loss of CIC is associated with chromosomal segregation defects in human and mouse} cells.

Motivated by the apparent dynamic association of CIC with chromosomes, and to explore the relationship between CIC function and the cell cycle, we performed IF assays on synchronized NHA-derived $C I C^{\mathrm{KO}}$ cell lines $^{27}$. We synchronized the parental NHA cell line and two of its derivative $C I C^{\mathrm{KO}}$ cell lines (A2 and H9) at G2/M phase using the reversible cyclindependent kinase 1 (CDK1) inhibitor RO-3306 ${ }^{28}$. The synchronized populations were then observed at $1 \mathrm{~h}$ after drug removal ("post-release"), corresponding approximately to metaphase; 1.5-2.5 $\mathrm{h}$ post-release, corresponding approximately to telophase and cytokinesis; and $3 \mathrm{~h}$ postrelease, corresponding approximately to $\mathrm{G} 1$ phase (Figure $1 \mathrm{~b}$ ). Compared to the parental $C I C^{\mathrm{WT}}$ cell line, the $C I C^{\mathrm{KO}}$ lines showed 2.2 - 2.5-fold increases in metaphase alignment defects $(\mathrm{p}<$ 0.05) and 2 - 2.3-fold increases in CS defects at telophase/cytokinesis ( $p<0.01$; Methods; Figure 1c-d). We confirmed these observations in unsynchronized HEK and two HEK-derived $C I C^{\mathrm{KO}}$ cell lines (Supplementary Figure 1).

To determine whether CIC deficiency was also associated with CS defects in vivo, we examined the appearance of mitotic chromosomes at the anaphase-to-telophase stages in a recently reported Cic conditional knockout mouse model $^{29}$. IF staining of brain sections from embryonic day 13.5 animals with forebrain-specific Cic knockout $\left(\mathrm{Cic}^{f l / f l} ; \mathrm{Fox} G 1^{\text {cre/+}}\right)$ and from their heterozygous controls $\left(\mathrm{Cic}^{\mathrm{fll}+} ; \mathrm{Fox} G 1^{\mathrm{cre} /+}\right)$ revealed that Cic-null cells in the mitotically active ventricular zone (VZ) displayed an increased frequency of either lagging chromosomes or micronuclei (average 2.5-fold increase, $\mathrm{p}<0.0001$; Figure 1e). Along with our observations from cultured NHA and HEK cells, these observations link CIC loss to defects in mammalian CS. 


\section{Loss of CIC in NHA cells is associated with copy number alterations and aneuploidy.}

CS defects such as those observed in our $C I C^{\mathrm{KO}}$ lines, including the presence of micronuclei and lagging chromosomes, have been linked to copy number alterations in daughter cells $^{30-32}$. To determine whether evidence of copy number defects could be observed in CICdeficient cells, we performed single-cell genome sequencing $\left(\mathrm{DLP}+{ }^{33}\right)$ on DNA libraries from the parental $C I C^{\mathrm{WT}}$ NHA line and NHA-derived $C I C^{\mathrm{KO}}$ lines (A2 and $\mathrm{H} 9$ described above, and additional lines B6 and H10). Overall, the majority (73\%; 1,015/1,391) of the cells we sequenced were triploid, thus defining the wild type ploidy status of the NHA lines (Figure 1f). An increase in ploidy status (i.e. tetraploidy, pentaploidy, or hexaploidy) was observed in two of the $C I C^{\mathrm{KO}}$ cell lines. 58\% (163/283) of the A2 cells and 39\% (113/287) of the B6 cells displayed increased ploidy status. We interpret these data to indicate that, in $C I C$ mutant cells, there is an enrichment of cells that fail to complete cytokinesis, resulting in genome duplication that yields $6 \mathrm{~N}$ or partial $6 \mathrm{~N}$ status, which then may lead to distinct cellular subpopulations with increased ploidy. We confirmed increases in ploidy using interphase FISH analysis to observe the arms of chromosomes 1 and 19. Notably, chromosomal arm ploidy ratios showed comparable distributions in the FISH and DLP+ data across all cell lines, indicating concordance between the two methods (Supplementary Figure 2).

We next analyzed the DLP+ data to reveal the frequency and distribution of copy number segments in individual cells, where segments were defined as adjacent bins with identical copy number and neutral copy number was defined as an individual cell's determined ploidy state (Methods). While the $C I C^{\mathrm{KO}}$ lines did not show consistent differences in the number of neutral segments or segments with copy number gain or loss compared to $C I C^{\mathrm{WT}}$ cells (Figure $1 \mathrm{~g}$ ), 
segments with copy number loss were consistently longer in the $C I C^{\mathrm{KO}}$ lines compared to the parental NHA line $(\mathrm{p}<0.05$; Figure $1 \mathrm{~h})$. Thus, while the $C I C^{\mathrm{KO}}$ lines did not appear to incur more losses than the parental $C I C^{\mathrm{WT}}$ line, the losses occurring in $C I C^{\mathrm{KO}}$ cells seemed to involve larger chromosomal segments. Together, these observations are consistent with the notion that $C I C^{\mathrm{KO}}$ cell lines display increased CIN: specifically, an increased proportion of the genome was subject to copy number loss in $C I C^{\mathrm{KO}}$ cells compared to $C I C^{\mathrm{WT}}$ cells, and results indicated that $C I C^{\mathrm{KO}}$ cells may be susceptible to events that lead to ploidy alterations.

\section{CIC-deficient cells display delayed mitotic progression and dysregulated CCNB1}

\section{expression.}

Given our observations that CIC loss was apparently associated with CS defects and CIN, we hypothesized that CIC loss might affect mitotic progression. To investigate this, we enumerated cells in pro-metaphase, metaphase and telophase/cytokinesis in synchronized $C I C^{\mathrm{WT}}$ and $C I C^{\mathrm{KO}}$ NHA lines at regular intervals following drug release (Methods). In the parental $C I C^{\mathrm{WT}}$ line and the $C I C^{\mathrm{KO}}$ lines (A2 and H9), similar percentages ( $\sim 17 \%$ and $\sim 14-15 \%$, respectively) of the cells entered pro-metaphase $0.5 \mathrm{~h}$ post-release (Figure $2 \mathrm{a}$ ). However, while $17 \%$ of the $C I C^{\mathrm{WT}}$ cells had entered metaphase $1 \mathrm{~h}$ post-release, only $4-6 \%$ of the $C I C^{\mathrm{KO}}$ cells $(\mathrm{p}<0.05)$ had entered metaphase at that time. Similarly, while $15 \%$ of the $C I C^{\mathrm{WT}}$ cells had entered telophase/cytokinesis $2.5 \mathrm{~h}$ post-release, only $2-4 \%$ of the $C I C^{\mathrm{KO}}$ cells $(\mathrm{p}<0.001)$ had entered telophase/cytokinesis at that time. These findings are consistent with the notion that, compared to $C I C^{\mathrm{WT}}$ cells, $C I C^{\mathrm{KO}}$ cells could enter pro-metaphase, but exhibited delayed progress through subsequent cell cycle phases. 
The accumulation of various mitotic cyclins at specific stages throughout mitosis is critical for proper cell cycle progression ${ }^{34}$. Given CIC's established function as a transcription factor, we hypothesized that the $\mathrm{CS}$ defects observed in $C I C^{\mathrm{KO}}$ cells may be associated with transcriptional dysregulation of key target genes. To measure gene expression in comparable populations of $C I C^{\mathrm{WT}}$ and $C I C^{\mathrm{KO}}$ cells, we performed reverse transcription followed by quantitative polymerase chain reaction (RT-qPCR) in unsynchronized cells (UN), synchronized cells enriched for G2/M ( $0 \mathrm{~h}$ post-release), and mitotic fractions collected by shake-off $0.5 \mathrm{~h}$ post-release (Methods), which contain $61-82 \%$ pro-metaphasic cells (referred to as PM; Supplementary Figure 3). $C C N B 1$, which under normal conditions shows mRNA accumulation at the G2/M transition and through mitosis ${ }^{35}$, showed significantly reduced abundance in the $C I C^{\mathrm{KO}}$ lines (A2 and H9) compared to the parental $C I C^{\mathrm{WT}}$ line in $\mathrm{G} 2 / \mathrm{M}$ and PM cells $(\mathrm{p}<0.05$; Figure $2 \mathrm{~b})$. The other cyclin genes tested did not show consistent changes in mRNA expression across both $C I C^{\mathrm{KO}}$ cell lines. We confirmed the decreased expression of CCNB1 protein and also observed increased expression of CCND1/2/3 proteins in synchronized and unsynchronized $C I C^{\mathrm{KO}}$ cells compared to $C I C^{\mathrm{WT}}$ cells (Supplementary Figure 3 ). Given that CCNB1 is a key regulator of mitotic progression, reduced CCNB1 through down-regulation of $C C N B 1$ mRNA expression in mitotic $C I C^{\mathrm{KO}}$ cells supports the notion that loss of CIC can result in delayed entry into metaphase.

\section{Loss of CIC is associated with dysregulated expression of mitotic kinases and regulators}

\section{of the spindle assembly checkpoint (SAC).}

Defects in the SAC, which acts at the pro-metaphase-to-metaphase transition, and defective cytokinesis have both been associated with CS defects and resulting aneuploidy ${ }^{36}$. Expression of mitotic kinases and genes regulating the SAC are tightly controlled, with mRNA accumulation 
beginning in S-phase and peak mRNA levels occurring at the G2/M transition and throughout mitosis $^{37}$. We thus hypothesized that mitotic kinases and regulators of the SAC might also display dysregulated expression in $C I C^{\mathrm{KO}}$ cells, contributing to the mitotic defects and aneuploidy we observed in these cells. To investigate this, we measured expression of the known CIC target genes ETV4/5, the SAC regulator $H M G A 1$, the mitotic checkpoint complex kinases $B U B 1 / 3$ and $M A D 2 L 1$, and the mitotic kinases $P L K 1 / 3$ and $A U R K A / B$ in $C I C^{\mathrm{WT}}$ and $C I C^{\mathrm{KO}} \mathrm{NHA}$ cells. As anticipated, mRNAs corresponding to the known CIC targets ETV4 and ETV5 exhibited increased abundance in the $C I C^{\mathrm{KO}}$ cell lines compared to the $C I C^{\mathrm{WT}}$ line, both in synchronized and unsynchronized cells $(\mathrm{p}<0.05$; Figure $2 \mathrm{c})$. The mRNA abundance of the SAC regulator $H M G A 1$ and the mitotic kinase $P L K 3$ were also significantly increased in both $C I C^{\mathrm{KO}}$ lines enriched for synchronized G2/M and PM cells ( $\mathrm{p}<0.05$; Figure $2 \mathrm{c})$. Conversely, mRNAs for the mitotic kinases $P L K 1, A U R K A 1$, and the mitotic checkpoint complex kinases $B U B$ lexhibited reduced abundance in $\mathrm{G} 2 / \mathrm{M}$ and $\mathrm{PM} C I C^{\mathrm{KO}}$ cells compared to $C I C^{\mathrm{WT}}$ cells ( $<<0.05$; Figure $2 \mathrm{~d}$ ). $M A D 2 L 1, A U R K B$, and $B U B 3$ did not show significant differences in mRNA abundance in either $C I C^{\mathrm{KO}}$ line compared to $C I C^{\mathrm{WT}}$ cells (Supplementary Figure 3). Overall, these results are compatible with the notion that CIC loss is associated with dysregulated mRNA expression of some genes involved in regulating transitions in the cell cycle between pro-metaphase, metaphase, and cytokinesis.

To confirm that these mRNA expression changes were a consequence of CIC loss, we performed a rescue experiment in which we re-introduced CIC constructs into $C I C^{\mathrm{KO}}$ cells and measured gene expression using RT-qPCR. Given that CIC exists in two isoforms - namely the long (CIC-L) and short (CIC-S) forms - and that we have previously shown these to have different patterns of distribution ${ }^{38}$, we re-introduced each isoform individually to examine their 
effects on gene expression (A2 $2^{\mathrm{F}-\mathrm{CIC}-\mathrm{S}}$ and $\mathrm{A} 2^{\mathrm{F}-\mathrm{CIC}-\mathrm{L}}$; Figure $3 \mathrm{a}$ ). ETV4 and HMGA1 exhibited reduced expression upon re-introduction of either F-CIC-S or F-CIC-L $(p<0.001$ and $p<0.01$, respectively; Figure 3b-c), presumably as a consequence of restoration of CIC-mediated repressor activity. ETV5 and $P L K 3$, on the other hand, were re-repressed only upon reintroduction of F-CIC-S ( $p<0.001$ and $p<0.05$, respectively). Taken together, these data are compatible with the notion that HMGA1 and PLK3 may be novel targets of CIC. Interestingly, while re-introduction of CIC-S did not affect the expression of genes that show reduced expression upon CIC loss in unsynchronized cells (Supplementary Figure 3), it did partially restore higher expression of the SAC regulators $P L K 1, A U R K A$, and $B U B 1(\mathrm{p}<0.05)$ and of the M-phase cyclin $C C N B 1$ ( $\mathrm{p}<0.01)$ in synchronized G2/M populations (Figure 3d). Only BUB1 also showed a significant difference in expression upon re-introduction of CIC-L. While this could be an indirect consequence of CIC re-introduction, these results also raise the possibility that CIC may function as a transcriptional activator acting on these genes. Together, these observations are consistent with the notion that CIC-S, which is found both in the cytoplasm and the nucleus ${ }^{38}$, plays a significant role in the regulation of mitotic genes. The reduced expression of $C C N B 1, A U R K A, B U B 1$, and PLK1 in $C I C^{\mathrm{KO}}$ but not $C I C^{\mathrm{WT}}$ cells is consistent with the notion that CIC loss leads to gene expression alterations that may delay mitotic progression, perhaps contributing to CIN.

To determine whether these novel candidate target genes might be direct targets of CIC regulation, we identified canonical CIC binding sites ${ }^{39}$ within their promoter regions (Methods; Supplementary Table 1) and performed targeted chromatin immunoprecipitation (ChIP) followed by qPCR for these sites in unsynchronized and synchronized ( $0 \mathrm{~h}$ post-release, $\mathrm{G} 2 / \mathrm{M})$ cells. Other than the sites in the promoter regions of the known CIC target genes ETV4 and ETV5 
(Figure 3e), none of the sites tested showed enrichment compared to a negative control region (NCR) and compared to $C I C^{\mathrm{KO}}$ cells (examples in Supplementary Figure 3), indicating that CIC did not directly bind to these sites. Additional primers were also designed to target regions lacking canonical CIC binding sites in the promoter or gene body of $H M G A 1$ and $P L K 3$ where CIC binding enrichment has previously been reported by ChIP in the HOG cell line ${ }^{40}$ (PLK3-6, PLK-7, HMGA1-11, and HMGA1-12, Supplementary Table 1 and Supplementary Figure 4). The two sites tested for $P L K 3$ and one of the sites tested for $H M G A 1$ showed significant enrichment compared to $C I C^{\mathrm{KO}}$ cells in the G2/M-enriched population $(>2$-fold difference, $\mathrm{p}<$ 0.05; Figure 3e). Taken together, these observations support the contention that PLK3 and $H M G A 1$ may be direct targets of CIC regulation through interaction with non-canonical binding sites within their gene bodies.

\section{Nuclear CIC interacts with core subunits of the SWI/SNF complex.}

To gain further insight into CIC's nuclear interaction network, we performed immunoprecipitation (IP) assays for endogenous CIC and N-terminal MYC-fused CIC-S (using a MYC antibody) in nuclear fractions purified from the HEK293 cell line (Methods; Supplementary Figure 5a). The immunoprecipitates were then characterized using liquid chromatography followed by tandem mass spectrometry (IP-MS). This yielded 53 candidate CIC interacting proteins that were identified in at least two of the four replicate IP-MS experiments (Supplementary Table 2), and the known interactors ATXN1L and ATXN2 ${ }^{41}$ each identified in one replicate (Figure 4a). To identify pathways and protein complexes that were enriched for candidate interactors, we performed enrichment analyses using Metascape ${ }^{42}$ and Genemania ${ }^{43}$ and identified RNA processing, RNA localization, and chromatin organization as the most 
significantly enriched processes (FDR $<0.05$; Supplementary Table 3 ). Given CIC's known function as a transcriptional regulator, the nine candidate interactors that were associated with the Gene Ontology (GO) biological process term “chromatin organization” (GO term 0006325; MKI67, L3MBTL3, MECP2, PBRM1, SMARCA2, SMARCC1, RUVBL1, SIN3A, and UTP3; Figure 4a, Supplementary Table 4) were of particular interest, along with the polymerase POLR2A. We thus sought to replicate these candidate interactions in nuclear fractions of the NHA cell line using a variation of the IP-MS technique in which 'trigger peptides ${ }^{44}$ (Methods; Supplementary Table 5) were used to enhance the sensitivity of the MS data for these proteins of interest, along with the known CIC interactor ATXN1L ${ }^{41}$ and ARID1A and ARID2, which are known interactors of SMARCA2, SMARCC1 and POLR2A (reviewed in references ${ }^{45,46}$ ). We were thus able to observe in NHA cells the interactions between CIC and these 14 proteins, and we also identified additional candidate interactors, including the SWI/SNF complex proteins ARID1A, ARID2, and SMARCA5 (Supplementary Table 4). Since dysregulation of core members of the SWI/SNF complex and proteins associated with them (e.g. SMARCA2 ${ }^{47}$, ARID1A ${ }^{48}$, and SIN3A ${ }^{49}$ ) has previously been linked to CS defects, we hypothesized that CIC's apparent interaction with members of the SWI/SNF complex might be related to its cell cyclerelated functions.

To further explore the relationships between CIC and SWI/SNF complex members, we first performed reciprocal IPs to validate the interactions between endogenous CIC and SMARCA2, ARID1A, SMARCC1, and POLR2A in the NHA line (Figure 4b-e), and between FCIC-S and SMARCA2 and ARID1A in HEK cells (Supplementary Figure 5). To identify common interactors between CIC and either SMARCA2 or ARID1A, we performed additional IP-MS experiments against ARID1A and SMARCA2 using whole-lysate preparations of NHA 
cells (Supplementary Figure 5). These experiments identified 13 (ARID1A IP) and 11

(SMARCA2 IP) interactors that overlapped with candidate CIC interactors (Supplementary

Table 4). In addition to CIC, ten proteins (ARID1A, ARID2, FMR1, MTA2, L3MBTL3, PBRM1, SIN3A, SMARCA2, SMARCC1, and POLR2A) were recurrently identified as common interactors in the IP experiments (i.e. they interacted with CIC, ARIDIA, and SMARCA2).

\section{Nuclear CIC and core components of the SWI/SNF complex show similar spatial and temporal distribution during the cell cycle.}

Given CIC's striking spatial redistribution over the course of the cell cycle, we reasoned that similar patterns of redistribution in candidate protein interactors might support a cooperative role in CS. Using IF co-localization assays, we observed a striking apparent co-localization of F-CICS and ARID1A, SMARCA2 (Figure 4f-g), and SMARCC1 (Supplementary Figure 6) in HEK ${ }^{\mathrm{F}-}$

CIC-S cells during early cytokinesis. These proteins also showed similar localization dynamics and co-localization with CIC over the course of the cell cycle. Interestingly, SIN3A did not localize with CIC at early cytokinesis but appeared to do so during interphase (Figure 4h). ARID2 appeared to surround decondensing chromosomes in late telophase, but seemed to co-localize with CIC only later at early cytokinesis (Supplementary Figure 6b).

To further explore the co-localization of CIC and core SWI/SNF proteins, we performed proximity ligation assays (PLAs ${ }^{50}$; Methods), which produces a read-out when antibodies targeting candidate protein interactors are within $40 \mathrm{nM}$ of each other. In $\mathrm{HEK}^{\mathrm{F}-\mathrm{CIC}-\mathrm{S}}$ cells, PLA signals were detectable during interphase when anti-FLAG antibodies (marking CIC expression) were used with antibodies targeting ARID1A, SMARCA2, SMARCC1, or ARID2 (Figure 5a). 
The greatest signal was observed for ARID1A, followed by SMARCA2, perhaps indicating that CIC interacts with these two proteins more frequently than the other SWI/SNF proteins tested. For all interactors tested, PLA signals were mostly observed in the nucleus during interphase and early cytokinesis, while during metaphase/telophase they were detected in cytoplasmic regions (example shown in Figure 5b for ARID1A). This is consistent with the IF co-localization observed for CIC, ARID1A, SMARCA2, and SMARCC1 over the course of the cell cycle, as described above. Our results thus indicate that CIC, ARID1A, SMARCA2, and SMARCC1 have similar spatial distributions in the nucleus over the course of the cell cycle, and that CIC is in close proximity to the SWI/SNF protein complex, with interactions peaking at early cytokinesis and at interphase. Our data are thus compatible with the notion that CIC functions with SWI/SNF complex proteins.

\section{Loss of CIC and ARID1A cooperatively affect chromosome segregation.}

Given that ARID1A and SMARCA2 are known to regulate $\mathrm{CS}^{47,48}$, we speculated that CIC might cooperate with these proteins to regulate CS. To test this hypothesis, we first performed siRNA knockdowns (KDs) of ARID1A and SMARCA2 in parental $C I C^{\mathrm{WT}}$ NHA cells and in isogenic $C I C^{\mathrm{KO}}$ lines and assessed cell viability using crystal violet assays. Interestingly, only $A R I D 1 A$ knockdown resulted in significantly lower viability in $C I C^{\mathrm{KO}}$ cells compared to $C I C^{\mathrm{WT}}$ cells ( $\mathrm{p}<0.0003$; Figure 6a, data not shown for $S M A R C A 2$ knockdown). This decrease in viability was associated with an increase in metaphase alignment defects in $C I C^{\mathrm{KO}} / A R I D 1 A^{\mathrm{KD}}$ cells compared to cells lacking either CIC or ARID1A $(\mathrm{p}<0.05$; Figure $6 \mathrm{~b})$, consistent with the notion that combined loss of CIC and ARID1A cooperatively increases CS defects to a degree sufficient to reduce cell viability. 
We next sought to determine whether the candidate CIC target genes involved in cell cycle and SAC regulation that we identified in this study were co-regulated by CIC and ARID1A. In $C I C^{\mathrm{WT}}$ cells, ARIDIA knockdown was associated with increased expression of the known CIC targets ETV4 and ETV5 ( $\mathrm{p}<0.05$ and $\mathrm{p}<0.00001$, respectively; Figure 6c-d). Interestingly, combined loss of CIC and ARID1A was associated with a significant increase in the expression of ETV5 ( $\mathrm{p}<0.0001$; Figure 6d) and PLK3 ( $\mathrm{p}<0.001$; Figure 6e) and lower expression of $C C N B 1(\mathrm{p}<0.05$; Figure $6 \mathrm{f})$ relative to CIC loss alone, indicating that the expression of these genes is cooperatively regulated by $\mathrm{CIC}$ and ARID1A. The mitotic regulator HMGA1 (Figure $6 \mathrm{~g}$ ) only showed increased expression in $C I C^{\mathrm{KO}}$ cells and did not show a further increase in expression upon $A R I D 1 A$ knockdown, indicating that its expression is not regulated in cooperative fashion.

Taken together, these results indicate that CIC can interact with members of the SWI/SNF complex and can cooperatively regulate genes involved in CS; furthermore, ARIDIA knockdown appears to cooperate with CIC deficiency to decrease cell viability, perhaps through an increase in CS defects.

\section{Discussion}

In this study, we use human cells and a mouse model to describe a novel role for mammalian CIC in regulating mitotic progression and chromosome stability. We show that CIC is dynamically re-distributed over the course of the cell cycle, and that its loss is associated with defects in metaphase alignment and CS both in vitro and in vivo. Using DLP+ and interphase FISH analysis of NHA-derived $C I C^{\mathrm{KO}}$ cells, we show that these defects are associated with CIN and alterations in ploidy. We propose that these phenotypes result, at least in part, from the 
transcriptional dysregulation of genes involved in cell cycle regulation, which we observed upon loss of CIC (Figure 7). For instance, overexpression of HMGA1 $1^{51,52}$ or PLK3 ${ }^{53}$, both of which our results supported as novel targets of CIC transcriptional repression, has been shown to induce mitotic arrest and apoptosis. Meanwhile, a decrease in CCNB1 levels, which we also observed in response to CIC loss, has been shown to contribute to polyploidization in response to DNA damage ${ }^{54}$.

We also show that CIC interacts with core members of the SWI/SNF complex, namely ARID1A, SMARCA2, and SMARCC1. This raises the intriguing possibility that CIC might function as a member of the SWI/SNF complex. Of these, ARID1A knockdown was found to cooperatively increase mitotic defects and reduce cell viability in $C I C^{\mathrm{KO}}$ cells. We show that CIC and ARID1A cooperatively regulate the expression of genes involved in mitotic regulation, implying that this activity may be at least partially responsible for the defects observed in cells lacking both functional proteins (Figure 7). The $A R I D I A$ and $C I C$ genes are located on chromosomal arms 1p36.11 and 19q13.2, respectively, and are thus both subject to loss of heterozygosity $(\mathrm{LOH})$ in $1 \mathrm{p} / 19 \mathrm{q}$ co-deleted $\mathrm{LGGs}^{2,3}$. Although $C I C$ is frequently mutated in these tumours and $A R I D 1 A$ mutations are common in other cancer types ${ }^{55,56}$, only $0.02 \%(3 / 169)$ of $1 \mathrm{p} / 19 \mathrm{q}$ co-deleted LGG cases from The Cancer Genome Atlas (TCGA) harbour mutations in these two genes ${ }^{6,7,57}$. This observation, together with our own results, indicates that the combined inactivation of CIC and ARID1A is associated with decreased cellular viability, and may not be compatible with proliferation of cancer cells. If the response we observed when both protein products are lost can be recapitulated in tumour contexts, such a synthetic lethal relationship could possibly be exploited in the context of therapy for tumours lacking either functional CIC or ARID1A. 


\section{Conclusions}

Our study establishes a novel role for CIC in cell cycle regulation and accurate CS and shows that loss of CIC is associated with a disruption in these processes, resulting in aneuploidy. We present evidence that these consequences are mediated through the transcriptional dysregulation of genes involved in cell cycle regulation and the SAC, including $C C N B 1$ and the proposed novel CIC targets $H M G A 1$ and $P L K 3$. We also uncovered a previously unappreciated relationship between CIC and the SWI/SNF complex comprised of cell cycle-dependent interactions, showing that they function together to regulate gene expression and ensure proper CS. These novel roles ascribed to CIC establish it as an important regulator of the cell cycle and provide a plausible explanation for its role as a tumour suppressor across a wide range of cancer contexts. 


\section{Acknowledgements}

We thank the DNA sequencing, library construction, and biospecimen core facilities at the BC Cancer Genome Sciences Centre for technical support. We thank Robyn Roscoe and Joanne Johnson for expert Project Management support. The authors thank Diane Trinh for helpful edits and comments on the manuscript and Dr. G. Dawson (The University of Chicago, Illinois, USA) for providing the HOG glioma cell line. MAM acknowledges the support of the Canadian Institutes of Health Research (FDN-143288); the Canada Research Chairs program and the BC Cancer Foundation (BCCF), and is especially grateful to Ms. Donna Anderson for her generosity in supporting this project. GBM acknowledges support from the BC Cancer Foundation.

\section{Author contributions}

MAM and SC conceived and designed experiments. JS, SYC and SC produced the reagents and tools required for the in vitro experiments. STA and JAC provided the mice brain material. JS, SYC, SDL, AL, AG, and SC performed most experiments. AM, CSH, and GBM performed and/or advised on the mass spectrometry experiments and CSH and SC analyzed the data. SA, SP, RC, and EL performed and/or advised on the DLP+ experiments, and RDC and VL analyzed and interpreted the data. AL and SY performed the FISH experiments, and AL and SC analyzed the data. SC wrote initial drafts and MAM and VL edited the manuscript. JAC, SAA, GBM, GJC, and SY provided ideas, discussion and edits to the manuscript. MAM provided oversight.

\section{Data availability}

The data that support the findings in this study are available from the corresponding author upon reasonable request. 


\section{Ethics statement}

Animal use was approved by the University of Calgary Animal Care Committee (protocol AC16-0266) in compliance with the Guidelines of the Canadian Council of Animal Care.

\section{Competing interests statement}

SY is on the advisory boards of Bayer AG and Pfizer Inc., and has received travel support from Foundation Medicine Inc. SAA is a co-founder of and consultant to Contextual Genomics Ltd and is on the advisory boards of Sangamo Biosciences and Repare Therapeutics. The remaining authors declare no competing interests. 


\section{References}

1. Haidar, A., Arekapudi, S., DeMattia, F., Abu-Isa, E. \& Kraut, M. High-grade undifferentiated small round cell sarcoma with $\mathrm{t}(4 ; 19)(q 35 ; q 13.1) \mathrm{CIC}$-DUX4 fusion: emerging entities of soft tissue tumors with unique histopathologic features--a case report and literature review. Am J Case Rep 16, 87-94 (2015).

2. Bettegowda, C. et al. Mutations in CIC and FUBP1 contribute to human oligodendroglioma. Science 333, 1453-5 (2011).

3. Yip, S. et al. Concurrent $\mathrm{CIC}$ mutations, IDH mutations, and $1 \mathrm{p} / 19 \mathrm{q}$ loss distinguish oligodendrogliomas from other cancers. J Pathol 226, 7-16 (2012).

4. The Cancer Genome Atlas Research Network. Comprehensive molecular characterization of gastric adenocarcinoma. Nature 513, 202-209 (2014).

5. The Cancer Genome Atlas Research Network. Integrated genomic characterization of endometrial carcinoma. Nature 497, 67-73 (2013).

6. Cerami, E. et al. The cBio cancer genomics portal: an open platform for exploring multidimensional cancer genomics data. Cancer Discov 2, 401-4 (2012).

7. Gao, J. et al. Integrative analysis of complex cancer genomics and clinical profiles using the cBioPortal. Sci Signal 6, pl1 (2013).

8. Choi, N. et al. miR-93/miR-106b/miR-375-CIC-CRABP1: a novel regulatory axis in prostate cancer progression. Oncotarget 6, 23533-47 (2015).

9. Okimoto, R.A. et al. Inactivation of Capicua drives cancer metastasis. Nat Genet 49, 87-96 (2017).

10. Simon-Carrasco, L. et al. Inactivation of Capicua in adult mice causes T-cell lymphoblastic lymphoma. Genes Dev (2017).

11. Tan, Q. et al. Loss of Capicua alters early $T$ cell development and predisposes mice to $T$ cell lymphoblastic leukemia/lymphoma. Proc Natl Acad Sci U S A (2018).

12. Chan, A.K. et al. Loss of CIC and FUBP1 expressions are potential markers of shorter time to recurrence in oligodendroglial tumors. Mod Pathol 27, 332-42 (2014).

13. Gleize, V. et al. CIC inactivating mutations identify aggressive subset of $1 \mathrm{p} 19 \mathrm{q}$ codeleted gliomas. Ann Neurol 78, 355-74 (2015).

14. Specht, K. et al. Distinct transcriptional signature and immunoprofile of CIC-DUX4 fusion-positive round cell tumors compared to EWSR1-rearranged Ewing sarcomas: further evidence toward distinct pathologic entities. Genes Chromosomes Cancer 53, 622-33 (2014).

15. Antonescu, C.R. et al. Sarcomas With CIC-rearrangements are a Distinct Pathologic Entity With Aggressive Outcome: A Clinicopathologic and Molecular Study of 115 Cases. Am J Surg Pathol (2017).

16. Liao, S. et al. A genetic interaction analysis identifies cancer drivers that modify EGFR dependency. Genes Dev 31, 184-196 (2017).

17. Wang, B. et al. ATXN1L, CIC, and ETS Transcription Factors Modulate Sensitivity to MAPK Pathway Inhibition. Cell Rep 18, 1543-1557 (2017).

18. Targa, A. \& Rancati, G. Cancer: a CINful evolution. Curr Opin Cell Biol 52, 136-144 (2018).

19. Eggert, U.S., Mitchison, T.J. \& Field, C.M. Animal cytokinesis: from parts list to mechanisms. Annu Rev Biochem 75, 543-66 (2006).

20. Mullins, J.M. \& Biesele, J.J. Cytokinetic activities in a human cell line: the midbody and intercellular bridge. Tissue Cell 5, 47-61 (1973). 
21. Brito, D.A. \& Rieder, C.L. Mitotic checkpoint slippage in humans occurs via cyclin B destruction in the presence of an active checkpoint. Curr Biol 16, 1194-200 (2006).

22. Rieder, C.L. \& Maiato, H. Stuck in division or passing through: what happens when cells cannot satisfy the spindle assembly checkpoint. Dev Cell 7, 637-51 (2004).

23. Daniels, M.J., Wang, Y., Lee, M. \& Venkitaraman, A.R. Abnormal cytokinesis in cells deficient in the breast cancer susceptibility protein BRCA2. Science 306, 876-9 (2004).

24. Ogle, B.M., Cascalho, M. \& Platt, J.L. Biological implications of cell fusion. Nat Rev Mol Cell Biol 6, 567-75 (2005).

25. Sonoda, Y. et al. Formation of intracranial tumors by genetically modified human astrocytes defines four pathways critical in the development of human anaplastic astrocytoma. Cancer Res 61, 4956-60 (2001).

26. Post, G.R. \& Dawson, G. Characterization of a cell line derived from a human oligodendroglioma. Mol Chem Neuropathol 16, 303-17 (1992).

27. LeBlanc, V.G. et al. Comparative transcriptome analysis of isogenic cell line models and primary cancers links capicua (CIC) loss to activation of the MAPK signalling cascade. J Pathol 242, 206220 (2017).

28. Vassilev, L.T. et al. Selective small-molecule inhibitor reveals critical mitotic functions of human CDK1. Proc Natl Acad Sci U S A 103, 10660-5 (2006).

29. Ahmad, S.T. et al. Regulation of neural stem cell fate by the transcriptional repressor Capicua. Preprint at https://www.biorxiv.org/content/early/2018/09/01/335984 (2018).

30. Forment, J.V., Kaidi, A. \& Jackson, S.P. Chromothripsis and cancer: causes and consequences of chromosome shattering. Nat Rev Cancer 12, 663-70 (2012).

31. Gorter de Vries, A.R., Pronk, J.T. \& Daran, J.G. Industrial Relevance of Chromosomal Copy Number Variation in Saccharomyces Yeasts. Appl Environ Microbio/ 83(2017).

32. Schukken, K.M. \& Foijer, F. CIN and Aneuploidy: Different Concepts, Different Consequences. Bioessays 40(2018).

33. Laks, E. et al. Resource: Scalable whole genome sequencing of 40,000 single cells identifies stochastic aneuploidies, genome replication states and clonal repertoires. Preprint at https://www.biorxiv.org/content/early/2018/09/13/411058 (2018).

34. Malumbres, M. \& Barbacid, M. Cell cycle, CDKs and cancer: a changing paradigm. Nat Rev Cancer 9, 153-66 (2009).

35. Wasner, M. et al. Cyclin B1 transcription is enhanced by the p300 coactivator and regulated during the cell cycle by a CHR-dependent repression mechanism. FEBS Lett 536, 66-70 (2003).

36. Ganem, N.J., Storchova, Z. \& Pellman, D. Tetraploidy, aneuploidy and cancer. Curr Opin Genet Dev 17, 157-62 (2007).

37. Schmit, T.L. \& Ahmad, N. Regulation of mitosis via mitotic kinases: new opportunities for cancer management. Mol Cancer Ther 6, 1920-31 (2007).

38. Chittaranjan, S. et al. Mutations in $\mathrm{CIC}$ and IDH1 cooperatively regulate 2-hydroxyglutarate levels and cell clonogenicity. Oncotarget 5, 7960-79 (2014).

39. Jimenez, G., Shvartsman, S.Y. \& Paroush, Z. The Capicua repressor--a general sensor of RTK signaling in development and disease. J Cell Sci 125, 1383-91 (2012).

40. Yang, R. et al. Cic Loss Promotes Gliomagenesis via Aberrant Neural Stem Cell Proliferation and Differentiation. Cancer Res 77, 6097-6108 (2017).

41. Bowman, A.B. et al. Duplication of Atxn1l suppresses SCA1 neuropathology by decreasing incorporation of polyglutamine-expanded ataxin-1 into native complexes. Nat Genet 39, 373-9 (2007). 
42. Tripathi, S. et al. Meta- and Orthogonal Integration of Influenza "OMICs" Data Defines a Role for UBR4 in Virus Budding. Cell Host Microbe 18, 723-35 (2015).

43. Warde-Farley, D. et al. The GeneMANIA prediction server: biological network integration for gene prioritization and predicting gene function. Nucleic Acids Res 38, W214-20 (2010).

44. Erickson, B.K. et al. A Strategy to Combine Sample Multiplexing with Targeted Proteomics Assays for High-Throughput Protein Signature Characterization. Mol Cell 65, 361-370 (2017).

45. Wilson, B.G. \& Roberts, C.W. SWI/SNF nucleosome remodellers and cancer. Nat Rev Cancer 11, 481-92 (2011).

46. Reisman, D., Glaros, S. \& Thompson, E.A. The SWI/SNF complex and cancer. Oncogene 28, 165368 (2009).

47. Coisy-Quivy, M. et al. Role for Brm in cell growth control. Cancer Res 66, 5069-76 (2006).

48. Dykhuizen, E.C. et al. BAF complexes facilitate decatenation of DNA by topoisomerase Ilalpha. Nature 497, 624-7 (2013).

49. David, G., Turner, G.M., Yao, Y., Protopopov, A. \& DePinho, R.A. mSin3-associated protein, $\mathrm{mSds} 3$, is essential for pericentric heterochromatin formation and chromosome segregation in mammalian cells. Genes Dev 17, 2396-405 (2003).

50. Soderberg, O. et al. Direct observation of individual endogenous protein complexes in situ by proximity ligation. Nat Methods 3, 995-1000 (2006).

51. Fedele, M. et al. Role of the high mobility group A proteins in human lipomas. Carcinogenesis 22, 1583-91 (2001).

52. Fedele, M. et al. Overexpression of proteins HMGA1 induces cell cycle deregulation and apoptosis in normal rat thyroid cells. Cancer Res 61, 4583-90 (2001).

53. Wang, Q. et al. Cell cycle arrest and apoptosis induced by human Polo-like kinase 3 is mediated through perturbation of microtubule integrity. Mol Cell Biol 22, 3450-9 (2002).

54. Nakayama, Y. \& Yamaguchi, N. Role of cyclin B1 levels in DNA damage and DNA damage-induced senescence. Int Rev Cell Mol Biol 305, 303-37 (2013).

55. Wiegand, K.C. et al. ARID1A mutations in endometriosis-associated ovarian carcinomas. $N$ Engl J Med 363, 1532-43 (2010).

56. Zang, Z.J. et al. Exome sequencing of gastric adenocarcinoma identifies recurrent somatic mutations in cell adhesion and chromatin remodeling genes. Nat Genet 44, 570-4 (2012).

57. Ceccarelli, M. et al. Molecular Profiling Reveals Biologically Discrete Subsets and Pathways of Progression in Diffuse Glioma. Cell 164, 550-63 (2016). 
bioRxiv preprint first posted online Jan. 29, 2019; doi: http://dx.doi.org/10.1101/533323. The copyright holder for this preprint (which was not peer-reviewed) is the author/funder, who has granted bioRxiv a license to display the preprint in perpetuity.

It is made available under a CC-BY-NC-ND 4.0 International license.

a
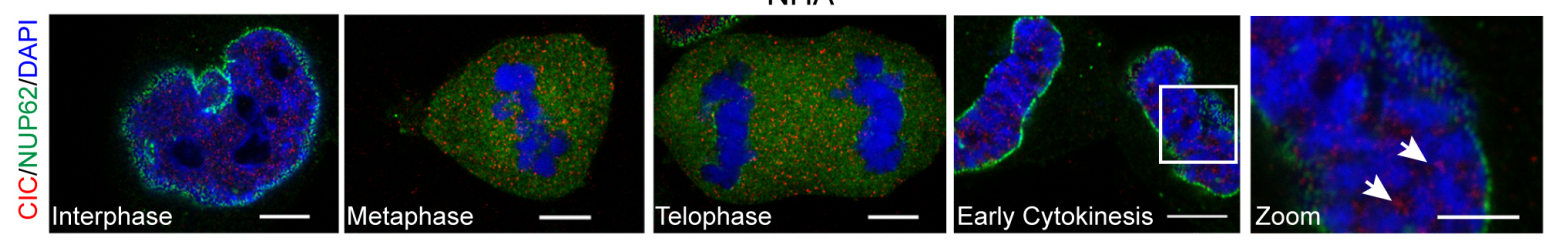

b

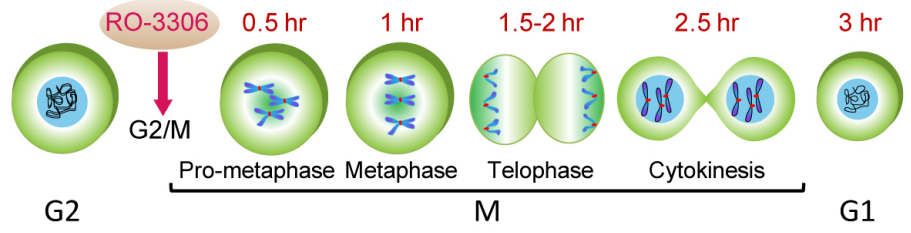

C

d
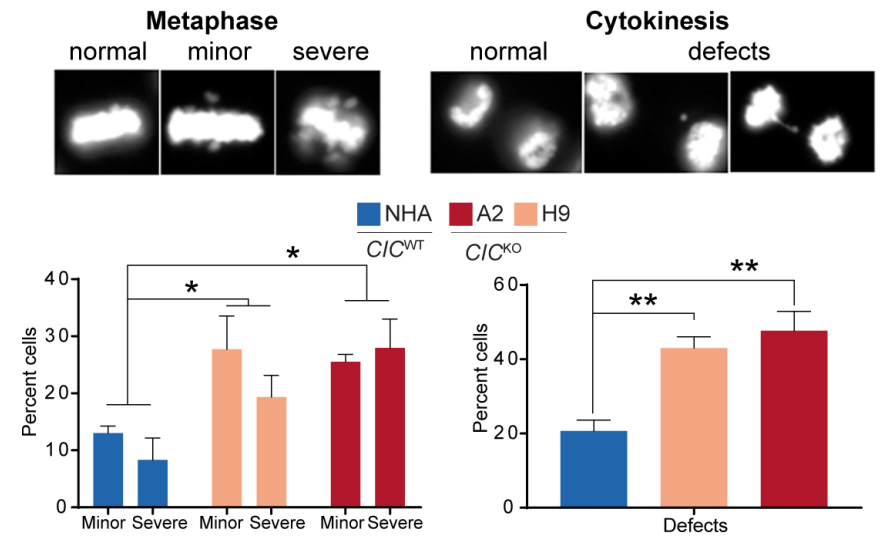

e
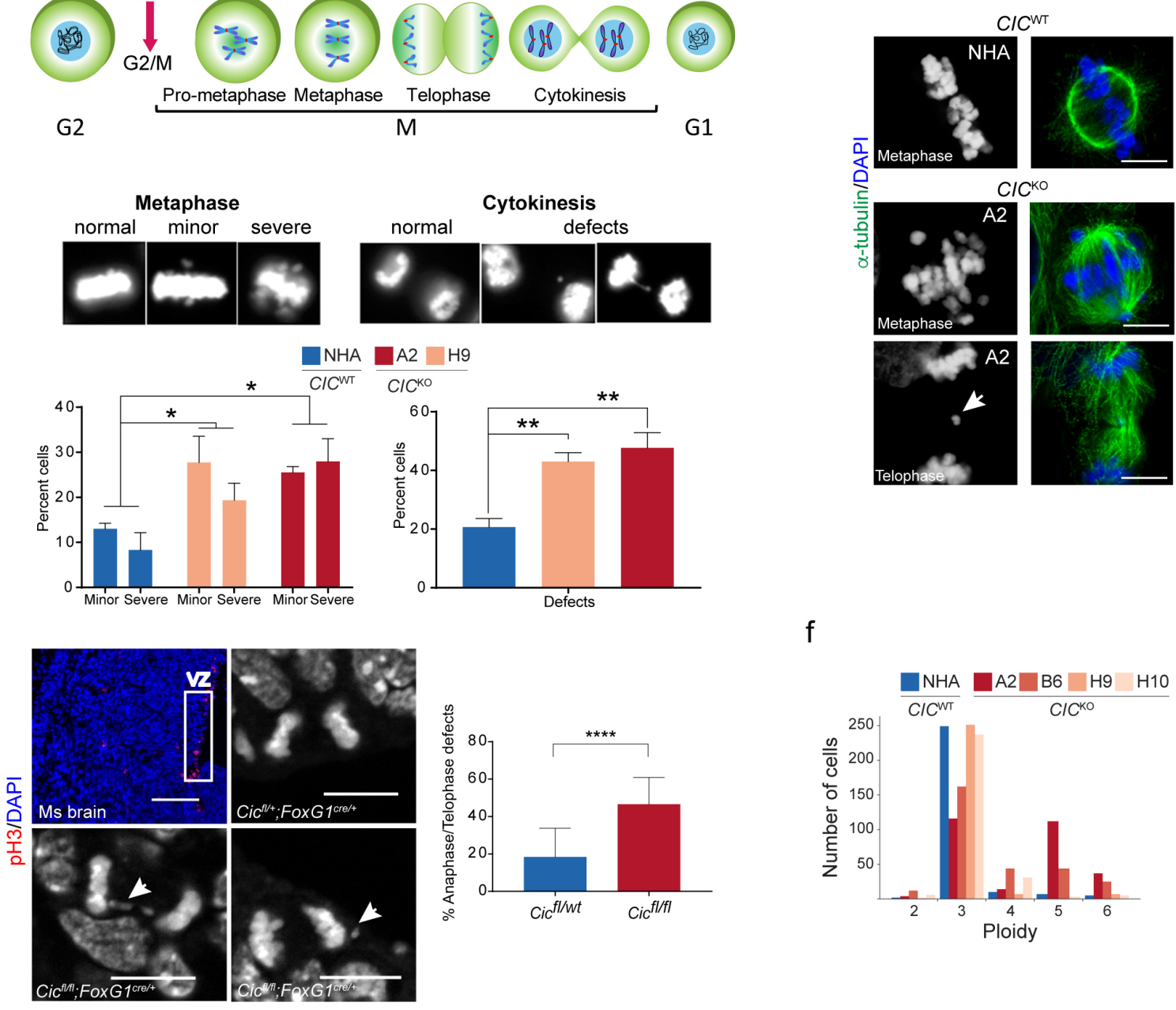

g

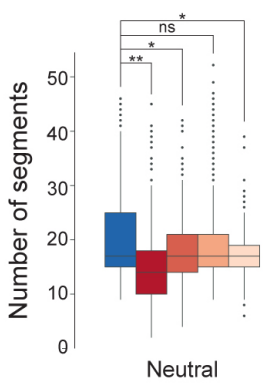

gain

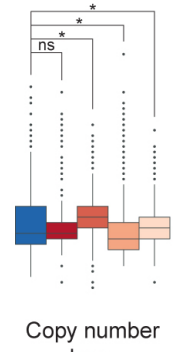

loss

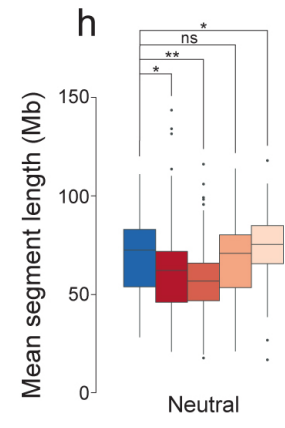

$* \mathrm{p}<5 \times 10^{-2}$ $* * p<5 \times 10^{-10}$ $* * * p<5 \times 10^{-20}$

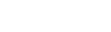

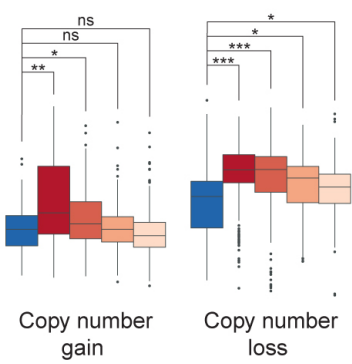

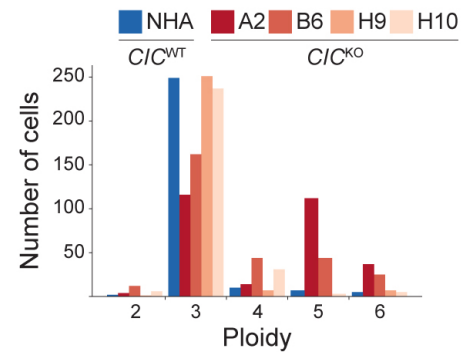




\section{Figure 1: Loss of CIC is associated with chromosomal segregation defects and CIN}

a. IF staining of endogenous CIC and the nuclear envelope protein NUP62 in the parental NHA line. CIC shows a punctate localization pattern (arrowheads) throughout the decondensing nucleus. Scale bars: $10 \mu \mathrm{m}$ and $5 \mu \mathrm{m}$ (zoomed image).

b. Time points at which cells were collected following synchronization with RO-3306.

c. Synchronized $C I C^{\mathrm{KO}}$ cells show defects in metaphase alignment and a lagging chromosome at telophase (arrowhead). Left: DAPI staining alone. Scale bars: $10 \mu \mathrm{m}$.

d. Top: example images of cells with normal or defective metaphase or cytokinesis. Bottom: proportions of cells assigned to each category. Bars represent the mean from three independent experiments and error bars indicate the standard error of the mean (s.e.m). $* p<0.05, * * p<0.01$ (two-sided Student's $t$-test comparing combined minor and severe defects).

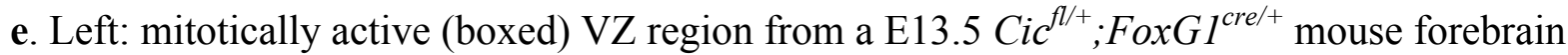
and representative images of lagging chromosomes and micronuclei (arrowheads, bottom left and right, respectively) in $C i c^{f l f l} ; F_{O x} G 1^{c r e /+}$ mice. Right: proportions of cells with defective anaphase/telophase. Bars represent the mean from four animals for each genotype and error bars indicate s.e.m $\left(\mathrm{n}=186\right.$ total for $\mathrm{Cic}^{f l /+} ; \mathrm{FoxG}^{\text {cre/+}}$ mice and 180 for $C i c^{f l / f l} ; \mathrm{Fox} G 1^{\text {cre/+ }}$ mice $)$. $* * * * \mathrm{p}<0.0001$ (Student's $t$-test). Scale bars: $100 \mu \mathrm{m}$ (top left) and $10 \mu \mathrm{m}$.

f. Number of cells with indicated ploidy status profiled by DLP+.

g-h. Tukey boxplots of segment counts (g) and mean length of segments (h) for indicated copy number statuses. ${ }^{*} \mathrm{p}<5 \times 10^{-2}, * * \mathrm{p}<5 \times 10^{-10}, * * * \mathrm{p}<5 \times 10^{-20}$ (Wilcoxon test with Bonferroni correction for each copy number status). 


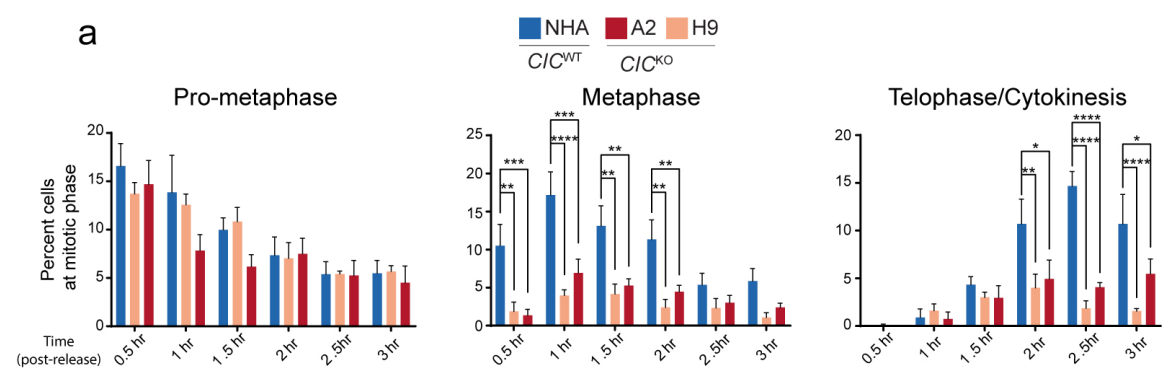

b

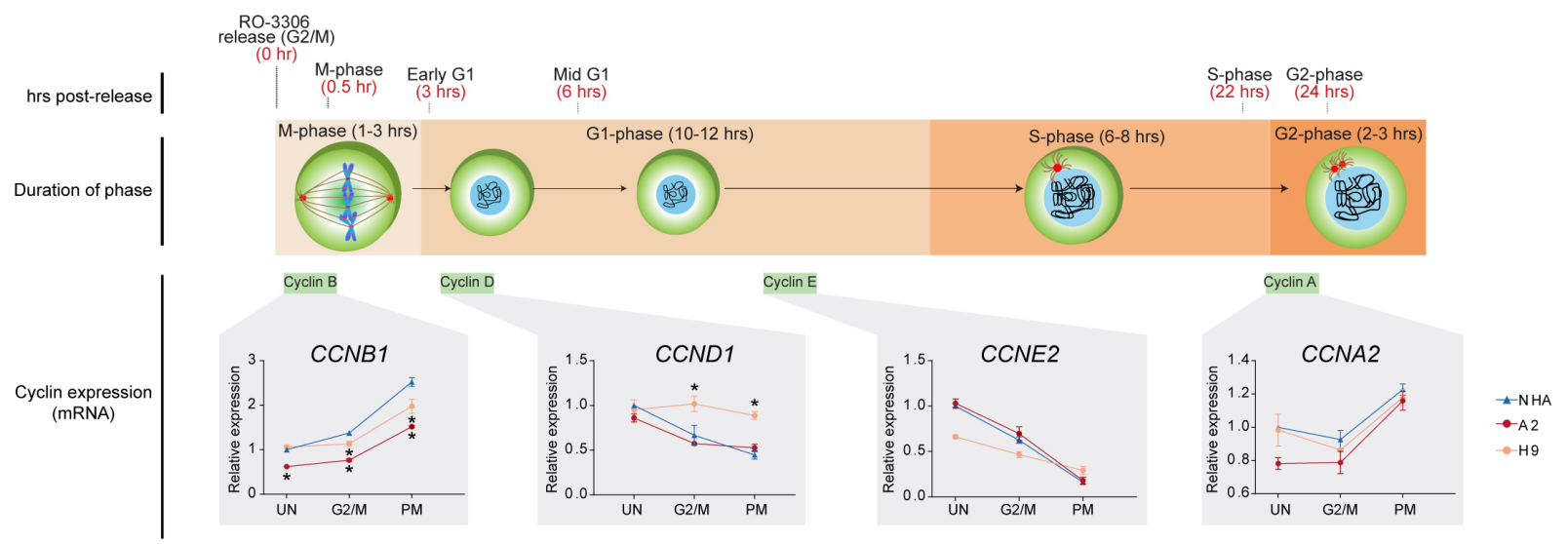

C
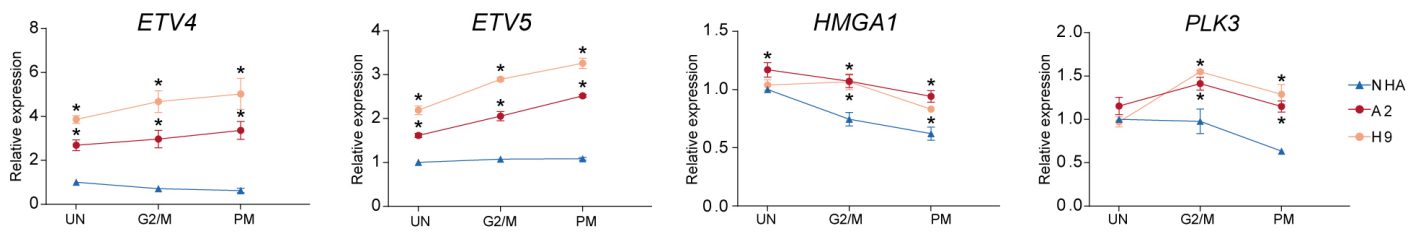

d
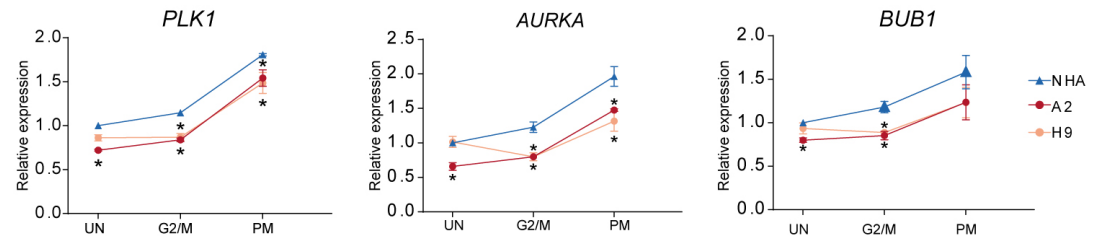

Figure 2. Loss of CIC is associated with transcriptional dysregulation of cell cycle regulators and members of the SAC complex.

a. Proportion of $C I C^{\mathrm{WT}}(\mathrm{NHA})$ and $C I C^{\mathrm{KO}}$ (A2 and H9) cells classified as being in prometaphase, metaphase, or telophase/cytokinesis based on DAPI and $\alpha$-tubulin staining (see examples in Figure 1a and c) at six time points following RO-3306 release. Bars represent the mean of at least 747 cells from three independent experiments, and error bars represent s.e.m. ${ }^{*} \mathrm{p}$ 
$<0.05, * * \mathrm{p}<0.01,{ }^{* * *} \mathrm{p}<0.001$ (one-way ANOVA with Holm-Sidak's multiple comparison test for each time point).

b-d. mRNA expression of $C C N B 1, C C N D 1, C C N E 2$, and $C C N A 2$ (b), the known CIC targets ETV4 and ETV5 and the mitotic regulators HMGA1 and PLK3 (c), and the mitotic kinases PLK1, $A U R K A 1$, and $B U B 1$ (d) in unsynchronized cells (UN), synchronized cells at $\mathrm{G} 2 / \mathrm{M}(0 \mathrm{~h}$ postrelease) and pro-metaphasic (PM) cells obtained by mitotic shake-off at $0.5 \mathrm{~h}$ post-release, as measured by RT-qPCR. Expression is shown as fold-changes relative to the unsynchronized NHA cells. Error bars indicate s.e.m. ${ }^{*} \mathrm{p}<0.05,{ }^{*} \mathrm{p}<0.01,{ }^{* *} \mathrm{p}<0.001$ (one-way ANOVA with Holm-Sidak's multiple comparison test for each time point). 
bioRxiv preprint first posted online Jan. 29, 2019; doi: http://dx.doi.org/10.1101/533323. The copyright holder for this preprint (which was not peer-reviewed) is the author/funder, who has granted bioRxiv a license to display the preprint in perpetuity.

It is made available under a CC-BY-NC-ND 4.0 International license.

a

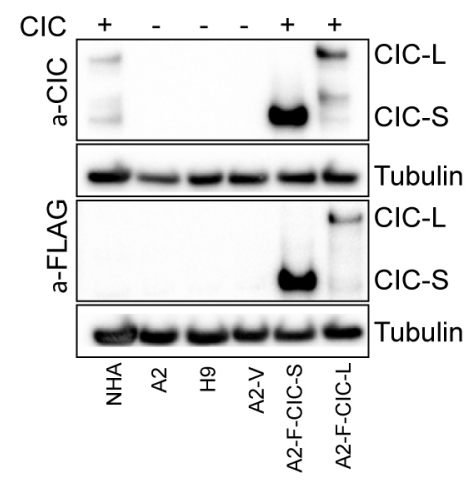

d

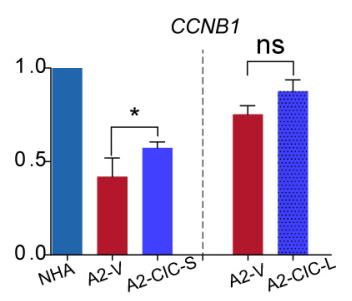

b
NHA $\left(C / C^{W T}\right)$
$\mathrm{A} 2-\mathrm{V}\left(\mathrm{Cl} \mathrm{C}^{\mathrm{K}}, \mathrm{vector}\right)$

F-CIC-S re-introduced

F-CIC-L re-introduced
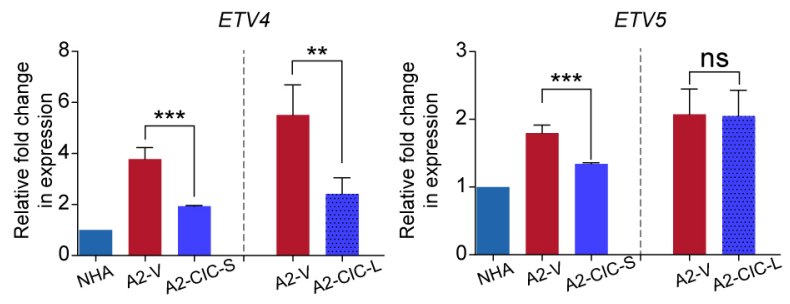

C

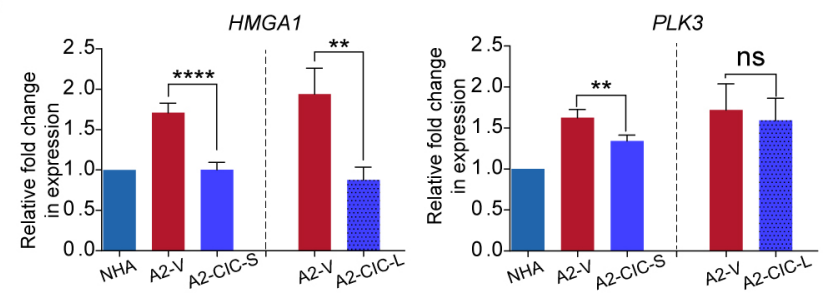

e

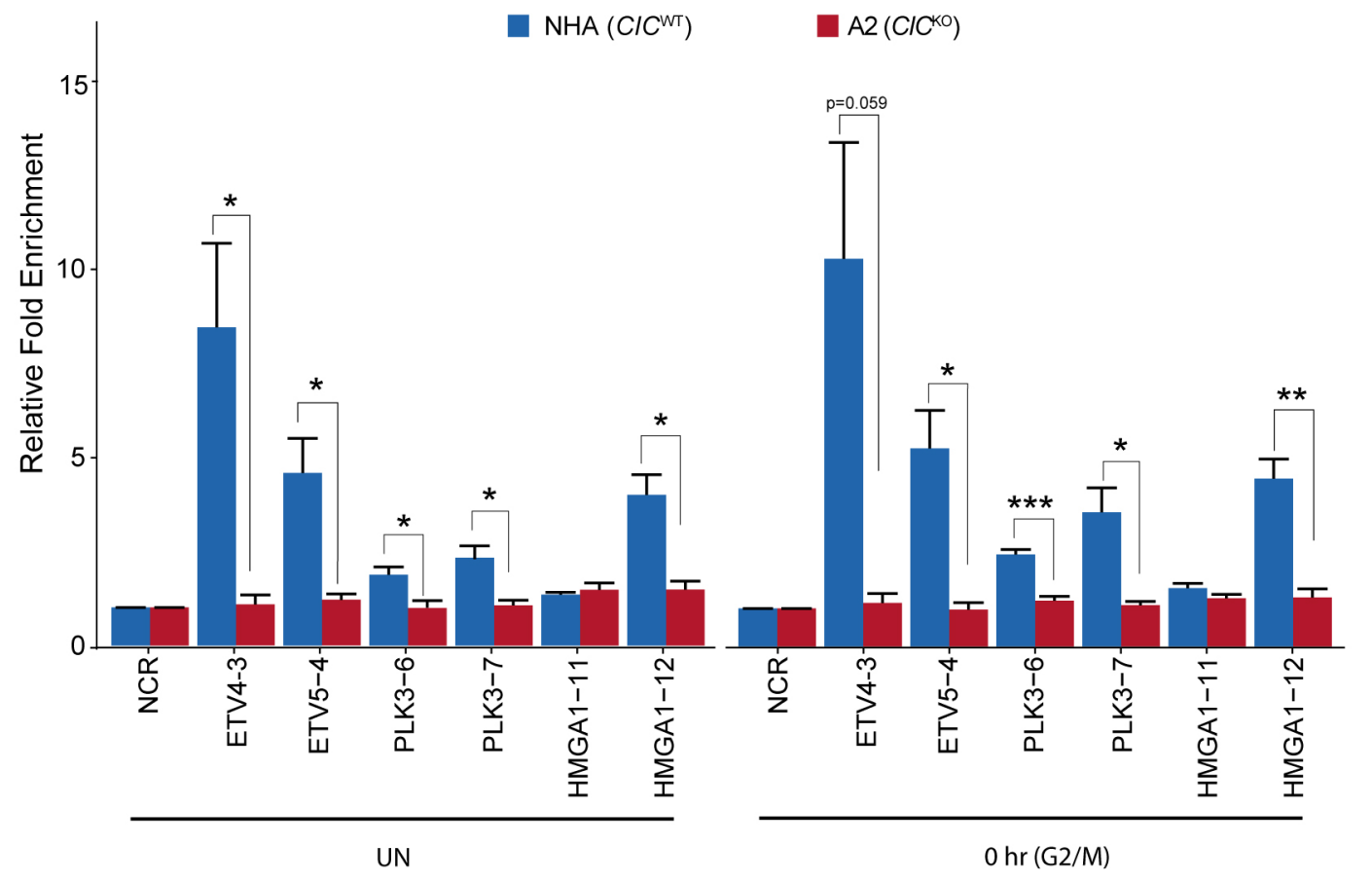


Figure 3. CIC regulates the expression of mitotic regulators.

a. Western blot showing expression of endogenous CIC-L and CIC-S in the parental NHA line $\left(C I C^{\mathrm{WT}}\right), C I C^{\mathrm{KO}}$ lines (A2 and $\left.\mathrm{H} 9\right)$, and A2 cells ectopically expressing an empty vector (V), FCIC-S, or F-CIC-L, detected using antibodies against CIC (top) or FLAG (bottom).

b-d. Relative expression of the known CIC targets ETV4 and ETV5 (b) and mitotic regulators showing increased (c) or decreased (d) expression in $C I C^{\mathrm{KO}}$ cells compared to $C I C^{\mathrm{WT}}$ cells. Gene expression in A2 cells expressing F-CIC-S or F-CIC-L was measured in independent experiments. Panels b and c represent measurements made in unsynchronized cells, while panel $\mathrm{d}$ represents measurements made in synchronized cells $0.5 \mathrm{~h}$ post-release. Expression is shown as fold-changes relative to the NHA cells. Bars represent the mean from three independent experiments and error bars indicate s.e.m. ${ }^{*} \mathrm{p}<0.05, * * \mathrm{p}<0.01, * * * \mathrm{p}<0.001, * * * * \mathrm{p}<0.0001$ (two-sided Student's $t$-test).

e. Enrichment of CIC binding at indicated sites relative to the NCR, as measured by ChIP-qPCR for $\mathrm{CIC}$ in $C I C^{\mathrm{WT}}$ (NHA) and $C I C^{\mathrm{KO}}$ (A2) cell lines in unsynchronized (UN) and synchronized $\mathrm{G} 2 / \mathrm{M}$ cells. Bars represent the mean from three independent experiments and error bars represent sem. ${ }^{*} \mathrm{p}<0.05$ (two-sided Student's $t$-test). 
a

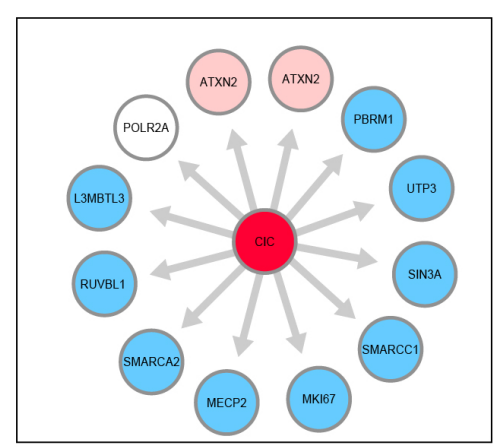

f
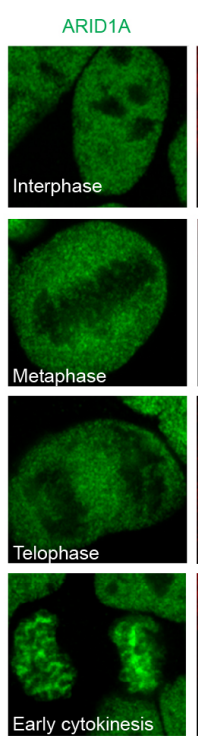

$\mathrm{h}$

h SIN3A
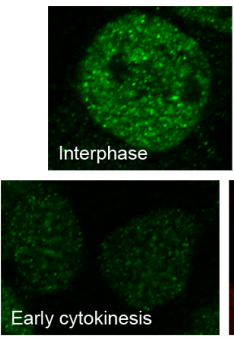

b

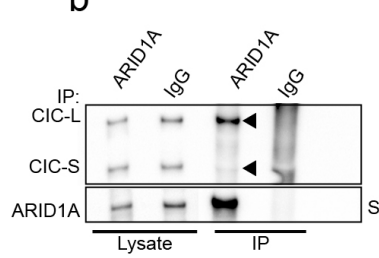

C

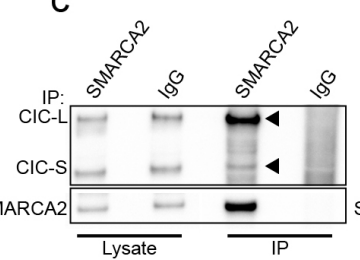

d

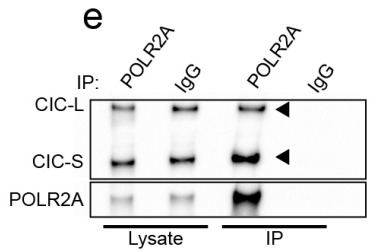

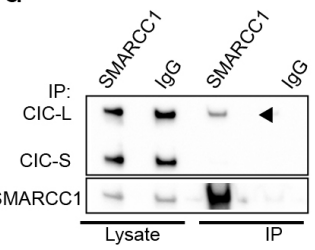

g

SMARCA2
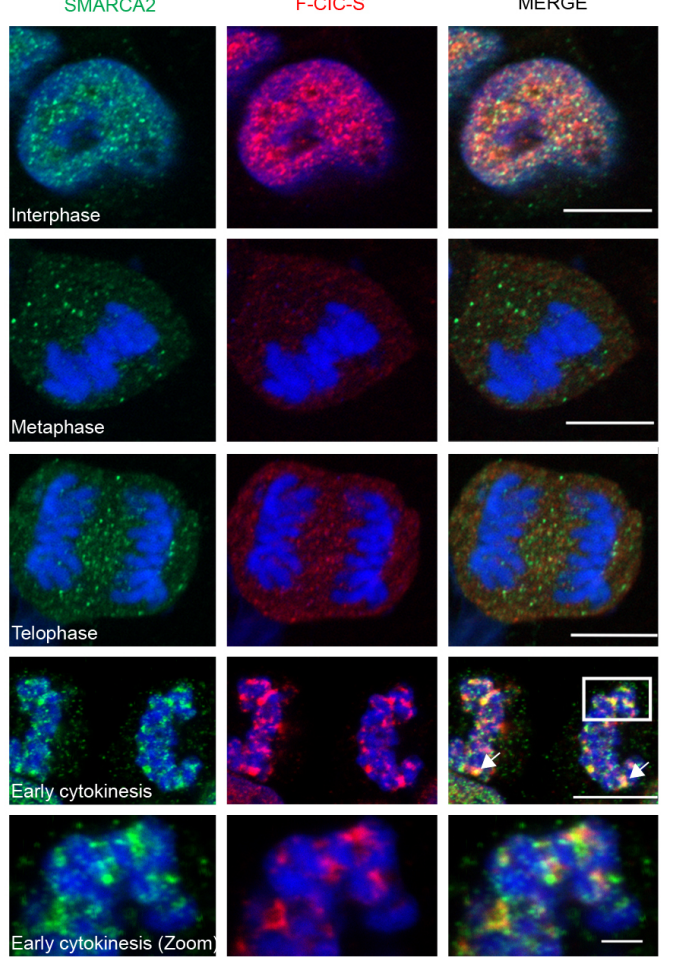

Figure 4: Nuclear CIC interacts with members of the SWI/SNF complex.

a. Interaction map showing select CIC interactors identified by IP-MS assays, namely the nine candidate CIC interactors that belong to the "Chromatin organization" GO term (blue), POLR2A (white), a known interactor of SMARCA2 and SMARCC1, and known CIC interactors (pink). 
b-e. Reciprocal IPs confirming the interaction between CIC and ARID1A (b), SMARCA2 (c), SMARCC1 (d), and POLR2A (e) in the parental NHA cell line, visualized by western blot. CICL and CIC-S bands are marked with arrowheads.

f-h. Localization of F-CIC-S (FLAG, red), ARID1A (f) SMARCA2 (g), and SIN3A (h, all green) in $\mathrm{HEK}^{\mathrm{F}-\mathrm{CIC}-\mathrm{S}}$ cells at indicated phases of the cell cycle, detected by IF. DNA was stained with DAPI (blue). Arrowheads indicate co-localization of CIC and the relevant interactor at early cytokinesis (yellow foci). Scale bars: $10 \mu \mathrm{m}$ and $5 \mu \mathrm{m}$ (zoomed image in $\mathrm{g}$ ). 
a
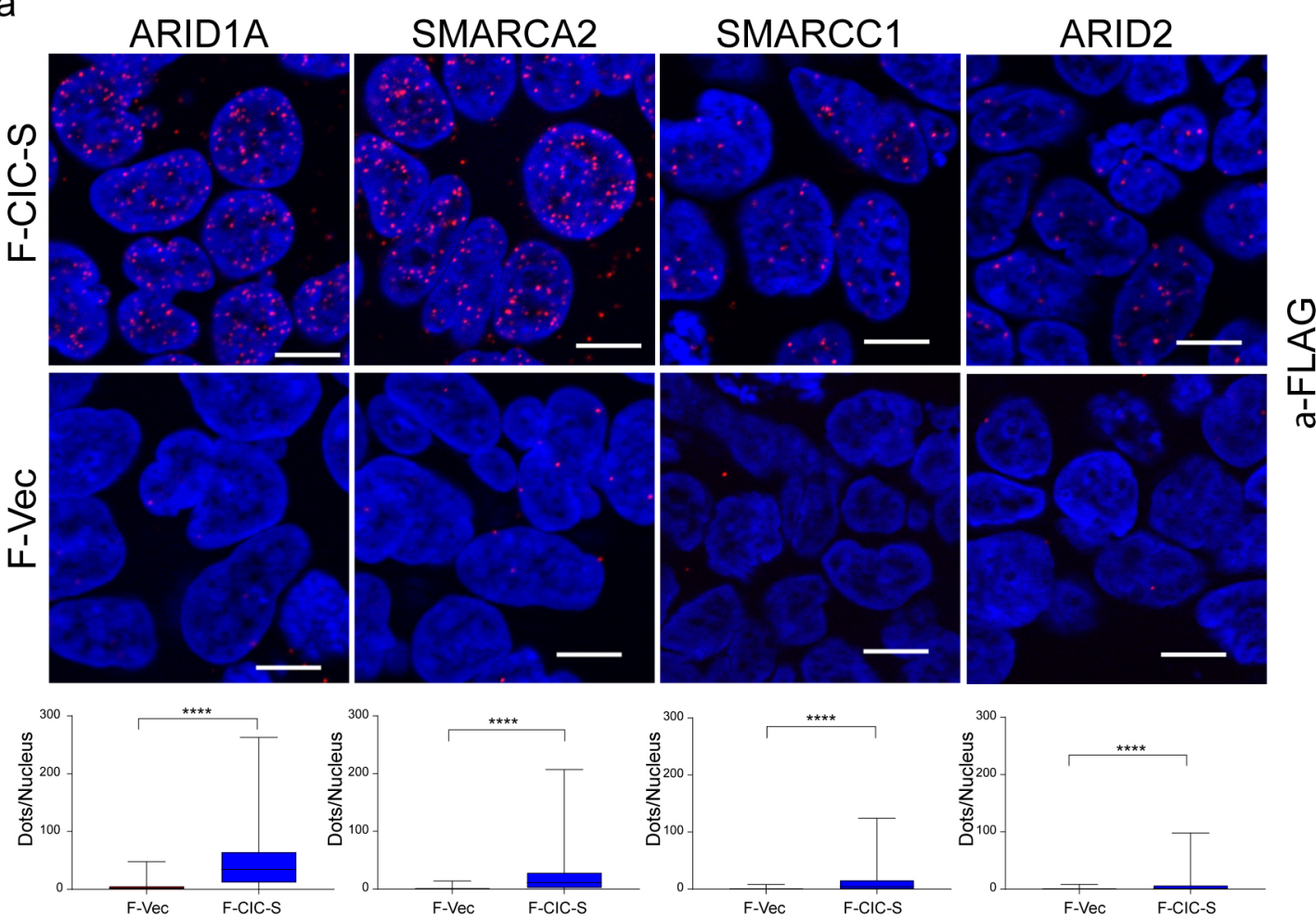

b

ARID1A

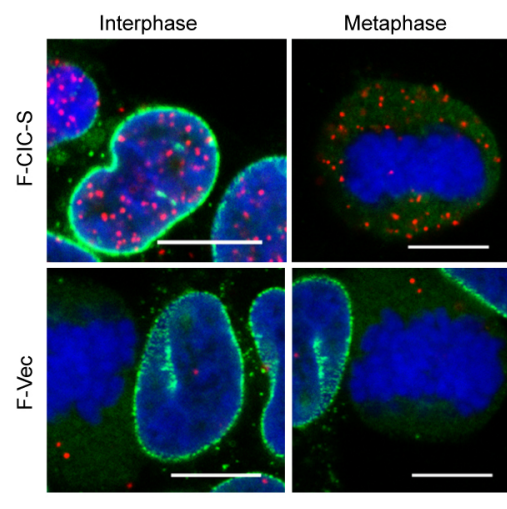

Telophase

Early cytokinesis

Metaphase

Early Cytokinesis
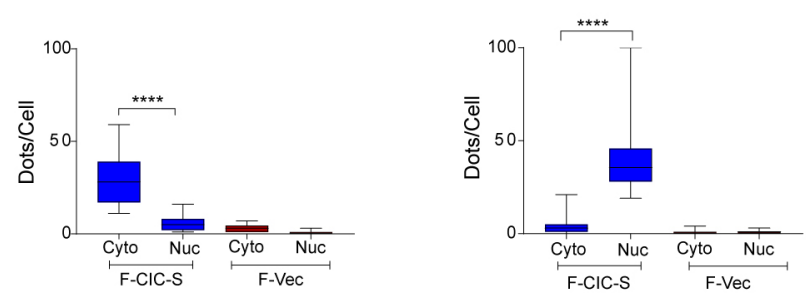
Figure 5: PLA assays indicate CIC and SWI/SNF complex proteins are in close proximity. a. Top: representative PLAs using antibodies against FLAG and ARID1A, SMARCA2, SMARCC1, or ARID2 in HEK-derived $C I C^{\mathrm{KO}}$ cells expressing F-CIC-S (HEK ${ }^{\mathrm{F}-\mathrm{CIC}-\mathrm{S}}$, top) or an empty vector $\left(\mathrm{HEK}^{\mathrm{F}-\mathrm{Vec}}\right.$, bottom). DNA was visualized using DAPI staining (blue), and PLA spots indicating close proximity of the proteins assayed $(<40 \mathrm{~nm})$ are visualized in red. Bottom: quantifications of PLA spots per nuclear region. The line represents the median of at least 532 cells, the hinges represent the first and third quartiles, and the error bars represent the total range. Scale bars: $10 \mu \mathrm{m}$. ****p $<0.0001$ (Welch's $t$-test).

b. Top: representative PLAs using antibodies against FLAG and ARID1A in HEK ${ }^{\text {F-CIC-S }}$ (top) and $\mathrm{HEK}^{\mathrm{F}-\mathrm{Vec}}$ (bottom) cells. Bottom: quantification of PLA spots in nuclear (Nuc) and cytoplasmic (Cyto) regions in cells at metaphase and early cytokinesis. The nuclear envelope was visualized by IF staining of NUP62 (green). In the box and whiskers plots, the hinges represent the first and third quartiles, the line represents the median, and the error bars represent range. Scale bars: $10 \mu \mathrm{m} . * * * * \mathrm{p}<0.0001$ (one-way ANOVA with Holm-Sidak's multiple comparison). 
a
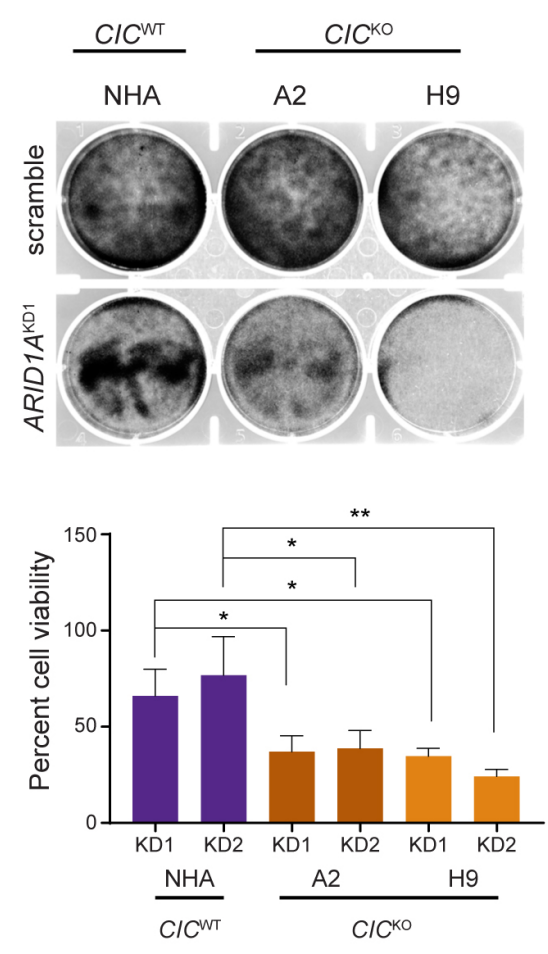

b

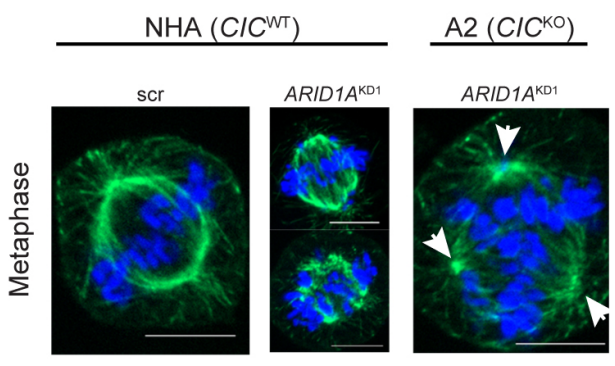

ARID1A $\mathrm{KD}^{\mathrm{D} 1}$
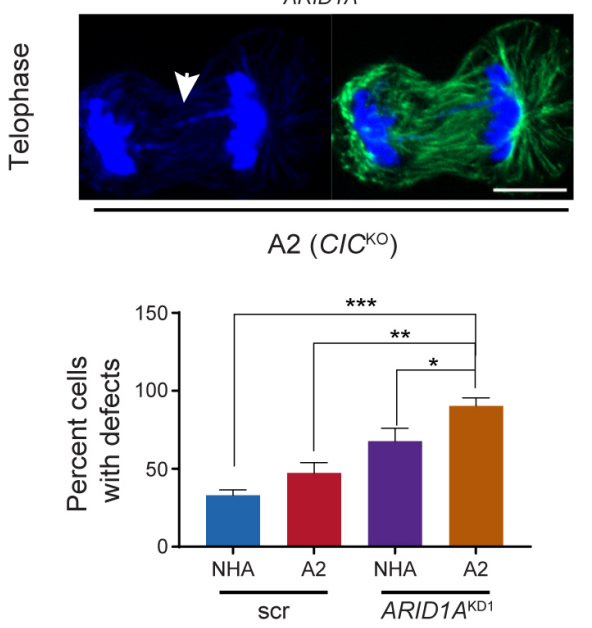

$\frac{\mathrm{NHA}, \mathrm{scr}}{\mathrm{NHA}, A R I D 1 A^{\mathrm{KT}}} \frac{\mathrm{A} 2, \mathrm{scr} \quad \mathrm{A} 2, A R I D 1 A^{\mathrm{KD}}}{\mathrm{Cl} / C^{\mathrm{KO}}}$

C

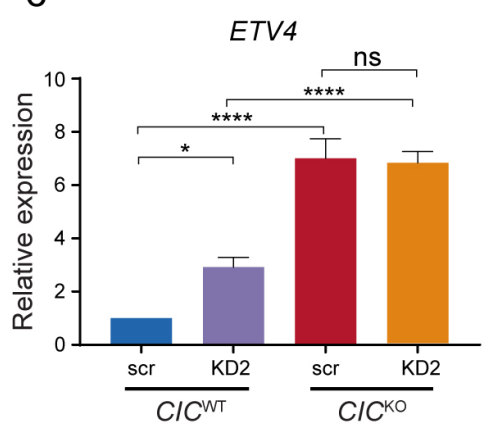

d

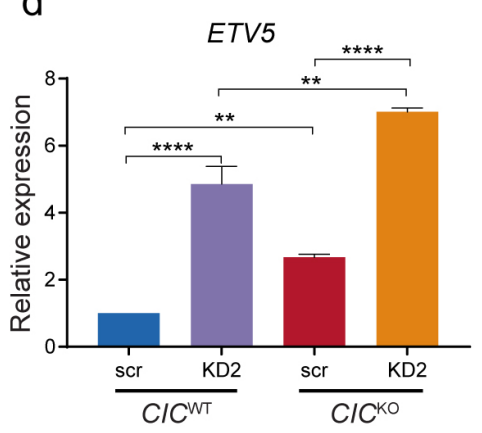

$\mathrm{e}$

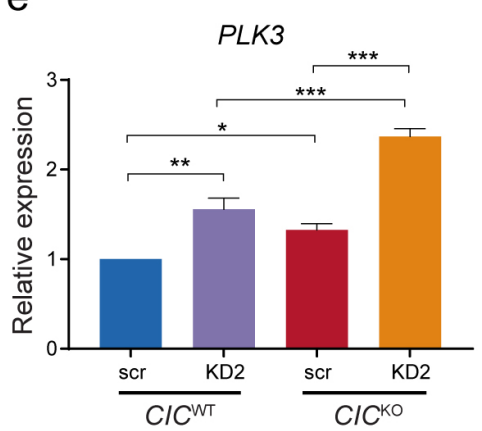

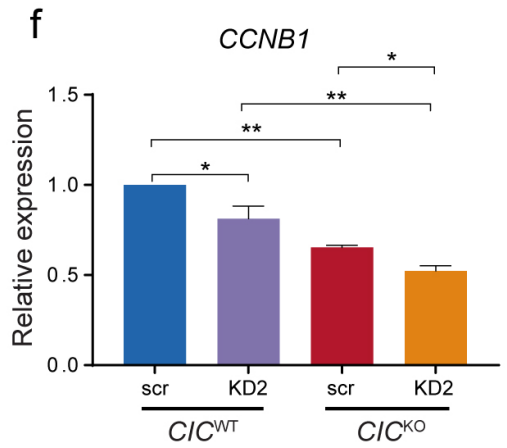

9

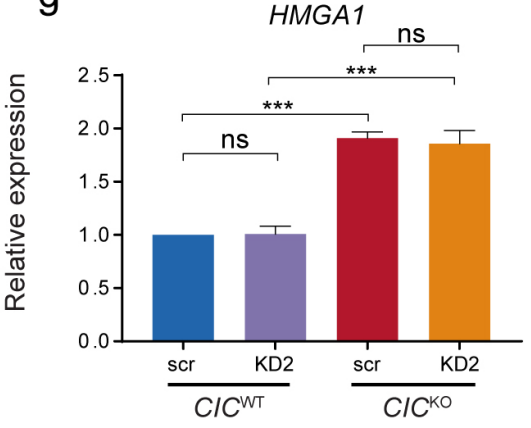




\section{Figure 6. Concurrent loss of CIC and ARID1A cooperatively increases mitotic defects and} decreases cell viability.

a. Crystal violet cell viability assay in $\mathrm{NHA}\left(C I C^{\mathrm{WT}}\right)$ and $\mathrm{A} 2$ and $\mathrm{H} 9\left(C I C^{\mathrm{KO}}\right)$ cells treated with a non-targeting siRNA (control, scramble) or one of two independent siRNAs against ARIDIA (KD1 and KD2, KD1 is shown in the representative image). Proliferation indices were generated relative to their respective controls, and quantifications from three independent experiments are shown below. Bars represent the mean and error bars indicate s.e.m. ${ }^{*} \mathrm{p}<0.05,{ }^{*} \mathrm{p}<0.01$ (oneway ANOVA with Holm-Sidak's multiple comparison).

b. Top: representative IF images of NHA $\left(C I C^{\mathrm{WT}}\right)$ and $\mathrm{A} 2\left(C I C^{\mathrm{KO}}\right)$ cells treated with a nontargeting siRNA (negative control, $\mathrm{NC} 1$ ) or an siRNA against $A R I D I A\left(A R I D 1 A^{\mathrm{KD} 1}\right)$. DNA (DAPI, blue) and expression of $\beta$-tubulin (green) were visualized at metaphase and telophase/cytokinesis. Arrowheads indicate multipolar chromosome alignments at metaphase and lagging chromosomes at cytokinesis in $C I C^{\mathrm{KO}}$ cells. Bottom: quantification of defects. Bars represent the mean across three independent experiments and error bars indicate s.e.m. Scale bars: $10 \mu \mathrm{m} .{ }^{*} \mathrm{p}<0.01$ (one-way ANOVA with Holm-Sidak's multiple comparison).

c-g. Relative expression of select genes in NHA $\left(C I C^{\mathrm{WT}}\right)$ and $\mathrm{A} 2\left(C I C^{\mathrm{KO}}\right)$ cells treated with a non-targeting control siRNA (scr) or a siRNA targeting ARID1A (KD2), as measured by RTqPCR. Expression is shown as fold-changes relative to the NHA cells treated with the nontargeting control siRNA. The known CIC targets ETV4 (c) and ETV5 (d) are shown, as are the mitotic regulators PLK3 (e), CCNB1 (f), and HMGA1 (g). *p $<0.05,{ }^{* *} \mathrm{p}<0.01,{ }^{* * *} \mathrm{p}<0.001$, $* * * * \mathrm{p}<0.0001$ ( $t$-tests with Holm-Sidak's multiple correction). 
a

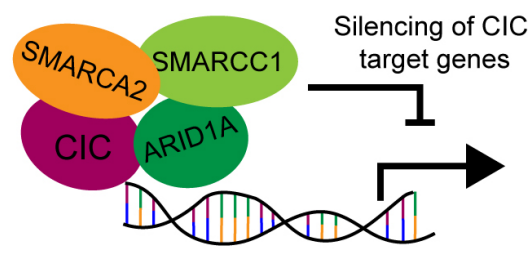

Normal mitosis

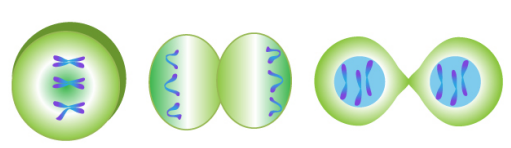

b

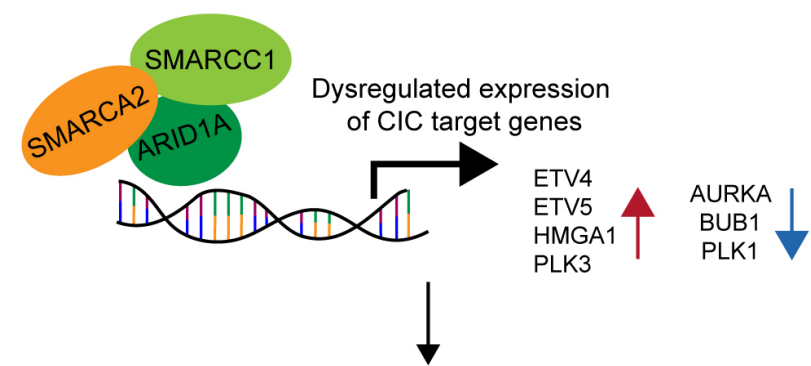

Defective mitosis, CIN, and aneuploidy

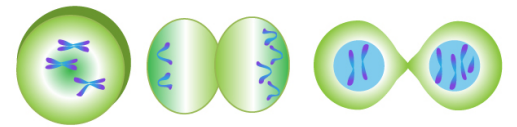

Figure 7. Working model: CIC functions as a tumour suppressor through regulation of CIN and ploidy alteration.

a. When CIC is present, it interacts with members of the SWI/SNF complex to regulate the expression of target genes, including mitotic regulators

b. In the absence of CIC, the known targets of CIC transcriptional regulation ETV4 and ETV5, as well as $H M G A 1$ and $P L K 3$, which our results indicate are novel direct targets of CIC regulation, show elevated expression. Mitotic regulators, including $A U R K A, B U B 1$, and PLK1 also show dysregulated expression that may be an indirect consequence of CIC loss. Together, these transcriptional changes appear to contribute to defective mitosis, CIN, and aneuploidy, which are observed at higher frequency in $C I C^{\mathrm{KO}}$ cells. 